05

\title{
Микромагнитное моделирование магнитоупругого эффекта в субмикронных структурах
}

\author{
() Р.В. Горев, О.Г. Удалов \\ Институт фоизики микроструктур РАН, \\ Нижний Новгород, Россия \\ E-mail: gorevrv@ipmras.ru
}

Поступила в Редакцию 15 апреля 2019 г.

В окончательной редакции 22 апреля 2019 г.

Принята к публикации 24 апреля 2019 г.

\begin{abstract}
Представлены результаты моделирования влияния деформаций на магнитные наночастицы эллиптической, квадратной и треугольной форм. Исследовано распределение намагниченности таких частиц в зависимости от их формы и приложенных деформаций. Показано, что деформация может приводить к появлению новых метастабильных состояний, а также к изменению типа основного состояния частицы. Деформация существенно изменяет распределение намагниченности частиц, что можно детектировать по их МСМконтрасту.
\end{abstract}

Ключевые слова: микромагнитное моделирование, магнитоупругий эффект, магнитно-силовая микроскопия, субмикронные структуры.

DOI: 10.21883/FTT.2019.09.48099.02N

\section{1. Введение}

В макроскопических масштабах существуют эффективные механизмы создания магнитного поля и управления магнитным состоянием системы с помощью электрических токов. Эти механизмы дают высокий КПД при преобразовании электрической энергии в магнитную. При уменьшении размера системы управление магнитным состоянием с помощью электрических токов становится крайне неэффективным. Для примера, в ячейках памяти на основе магнитного туннельного контакта КПД процесса перемагничивания магнитной частицы (записи информации) при пропускании тока составляет всего $5 \cdot 10^{-4 \%}[1]$. Данная проблема не может быть решена техническим (инженерным) путем. Поэтому многие научные группы в настоящее время исследуют фундаментально новые механизмы взаимодействия между электрическими и магнитными степенями свободы (магнитоэлектрический эффект). Особое внимание уделяется механизмам, в которых управление магнитными объектами осуществляется не электрическими токами, а электрическими полями [2-7]. Магнитоэлектрический (МЭ) эффект возникает, например, из-за взаимодействия Дзялошинского-Мория в геликоидальных магнетиках. Поверхностные МЭ явления возникают за счет эффекта поля в разбавленных магнитных полупроводниках и магнитных оксидах.

В последнее время активно исследуются гибридные структуры сегнетоэлектрик/ферромагнетик [8-16]. МЭ эффект в таких структурах возникает за счет комбинации магнитоупругого и электрострикционного эффектов. Стрикционный механизм можно считать объемным, несмотря на то, что деформации передаются через интерфейс.
Используя деформационный механизм МЭ эффекта, можно управлять магнитными частицами и переключаться между различными состояниями в таких частицах [17-23]. В силу симметрии стрикционный механизм не позволяет переключать намагниченность на противоположную. Он вызывает изменение направления оси анизотропии. Интересным при этом является исследование возможных типов переходов. Например, возможно ли за счет стрикционного МЭ эффекта переключение между двумя квазиоднородными состояниями, ориентированными ортогонально, или между вихревым и квазиоднородным состояниями.

Для изучения этих вопросов мы провели моделирование субмикронных магнитных частиц Ni. Исследовались основное и метастабильные состояния таких частиц в зависимости от однородных одноосных деформаций в них. При этом были рассмотрены различные размеры таких частиц и форма. Это позволило оценить параметры частиц, в которых деформации позволяют переключаться между различными типами магнитных состояний.

\section{2. Процедура моделирования}

Влияние деформаций моделировалось в пакете OOMMF [24] путем введения добавочной одноосной анизотропии $\left(K=8 \cdot 10^{3} \mathrm{~J} / \mathrm{m}^{3}\right)$ в плоскости магнитной пленки. Такая анизотропия возникает в пленках $\mathrm{Ni}$ вдоль оси однородного одноосного сжатия или растяжения (как при сгибании подложки, на которой находятся магнитные частицы, например), или при двухосных деформациях в плоскости (вдоль одной оси сжатие, а вдоль другой растяжение, как в частицах на поверхности 


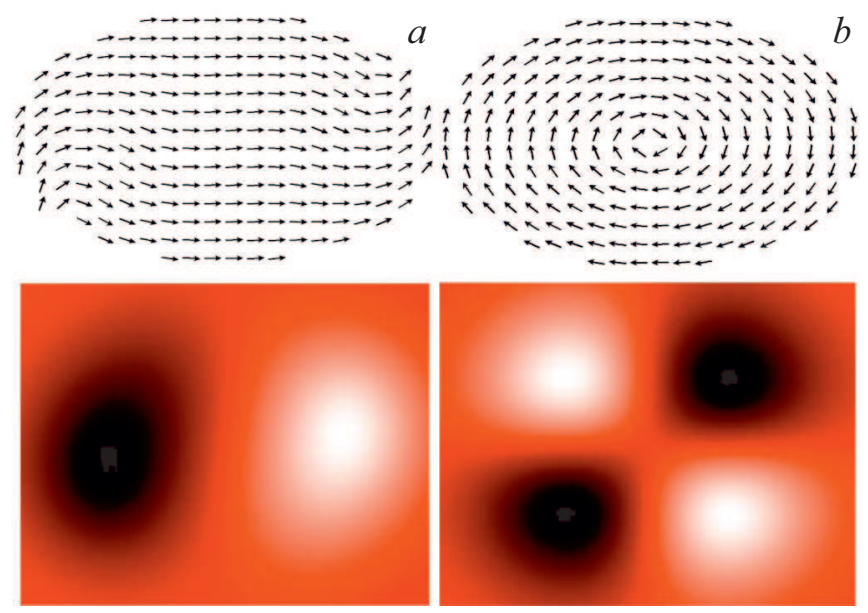

Рис. 1. Модельные распределения намагниченности и соответствующие им МСМ изображения Ni эллиптической частицы толщиной $30 \mathrm{~nm}$ с осями 1 и $0.5 \mu \mathrm{m}$ в квазиоднородном $(a)$ и одновихревом $(b)$ состояниях в отсутствие деформаций.

кристалла PMN-PT (011)). Величина наведенной анизотропии оценена исходя из величины константы магнитострикции $\mathrm{Ni}\left(-35 \cdot 10^{-6}\right)$ и оценки возможных деформаций порядка $0.2 \%$. Анизотропия может возникать за счет электрострикционного эффекта в сегнетоэлектриках или при изгибе подложки (например, стеклянной). Для моделирования были выбраны следующие параметры: намагниченность насыщения $M_{s}=4.9 \cdot 10^{5} \mathrm{~A} / \mathrm{m}$, обменная константа $A=9 \cdot 10^{-12} \mathrm{~J} / \mathrm{m}$, размер элементарной ячейки $5 \times 5 \times 30 \mathrm{~nm}$.

Для частиц в различных состояниях рассчитывались контраст, получаемый в магнитно-силовом микроскопе
(МСМ-контраст), и полная магнитная энергия, включающая в себя магнитостатическую энергию, энергию анизотропии и обменную энергию. При расчете МСМконтраста зонд считался точечным диполем, расположенным на высоте $600 \mathrm{~nm}$, а поле образца рассчитывалось как сумма дипольных полей от каждой элементарной ячейки. Размеры области, в которой рассчитывался МСМ-сигнал, превышали размеры образца на $200 \mathrm{~nm}$ с каждой стороны. В конечном счете, МСМ контраст рассчитывался как $z$-компонента градиента силы, действующей на зонд со стороны образца [25].

\section{3. Результаты и обсуждение}

Моделирование проводилось для частиц эллиптической (соотношение осей 1 к 2, длина большой полуоси от $200 \mathrm{~nm}$ до $1 \mu \mathrm{m}$ ), квадратной (длина стороны от $200 \mathrm{~nm}$ до $1 \mu \mathrm{m}$ ) и треугольной (длина каждой из сторон от $200 \mathrm{~nm}$ до $1 \mu \mathrm{m})$ формы толщиной $30 \mathrm{~nm}$.

\section{1. Эллиптические частицы}

Эллиптическая частица в отсутствие деформаций может находиться в квазиоднородном или одновихревом состоянии (рис. 1). В этом случае одновихревое состояние является основным (состояние с наименьшей энергией), а квазиоднородное - метастабильным. Для такой частицы существует два выделенных направления, по которым ее можно деформировать - вдоль ее длинной оси и вдоль короткой. При сжатии вдоль длинной (из-за отрицательной константы магнитострикции Ni дополнительная анизотропия появляется именно вдоль направления сжатия) оси количество возможных состоя-
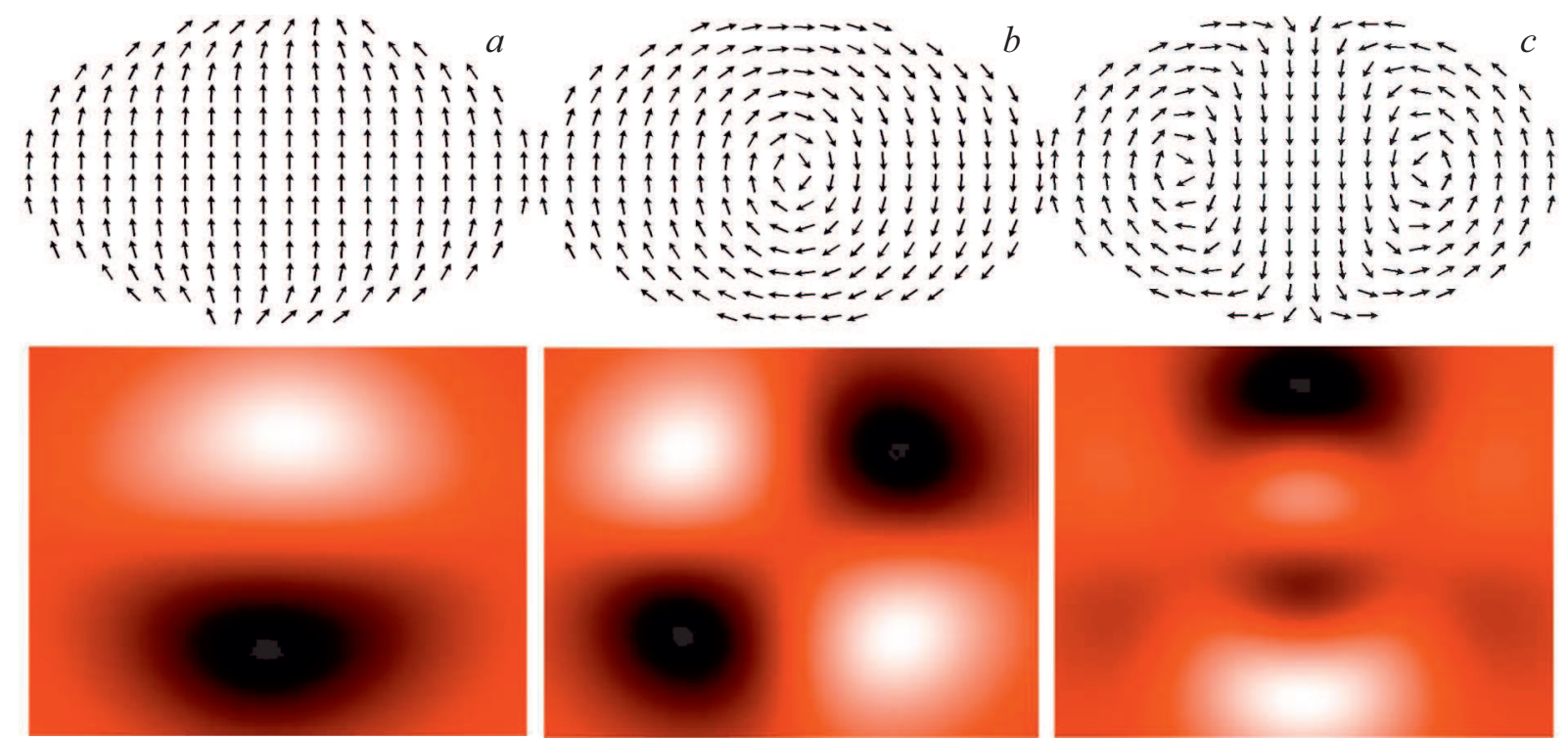

Рис. 2. Модельные распределения намагниченности и соответствующие им МСМ изображения Ni эллиптической частицы толщиной $30 \mathrm{~nm}$ с осями 1 и $0.5 \mu \mathrm{m}$ в квазиоднородном $(a)$, одновихревом $(b)$ и двувихревом $(c)$ состояниях при сжатии вдоль короткой оси эллипса. 
Таблица 1. Энергии различных состояний для недеформированной $\mathrm{Ni}$ эллиптической частицы с аспектным соотношением 2 к 1 и толщиной $30 \mathrm{~nm}$. (Подчеркнуто и выделено жирным значение энергии основного состояния)

\begin{tabular}{c|c|c}
\hline \multirow{2}{*}{$\begin{array}{c}\text { Длинная ось } \\
\text { эллипса, } \mu \mathrm{m}\end{array}$} & \multicolumn{2}{|c}{ Энергия состояния, $10^{-17}, J$} \\
\cline { 1 - 3 } & $\begin{array}{c}\text { квазиоднородное } \\
\text { (длинная ось) }\end{array}$ & одновихревое \\
\hline 1.0 & 8.3 & $\underline{\mathbf{7 . 0}}$ \\
0.8 & 6.1 & $\underline{\mathbf{5 . 1}}$ \\
0.6 & 4.1 & $\underline{\underline{\mathbf{2 . 5}}}$ \\
0.4 & 2.3 & $\underline{\underline{\mathbf{1 . 0}}}$ \\
0.2 & 0.9 &
\end{tabular}

ний не изменяется, но квазиоднородное состояние становится основным. Если же сжатие осуществляется вдоль короткой оси, то число возможных магнитных состояний увеличивается до трех (рис. 2). Деформированная частица в квазиоднородном состоянии намагничена вдоль короткой оси, а не длинной (рис. 2,a). Распределение намагниченности в одновихревом состоянии (рис. 2, $b$ ) несколько изменяется после деформации - область вихря вытягивается вдоль короткой оси, но на МСМ изображении это отражается слабо. Новое метастабильное состояние, появляющееся при сжатии вдоль короткой оси, - двухвихревое распределение намагниченности (рис. 2,c). Наибольший вклад в МСМ изображение от него будет давать однородно намагниченная область, расположенная между вихрями.

Важным вопросом является возможность перехода между различными состояниями за счет деформаций. Для изучения этого вопроса мы определяли полную энергию основных и метастабильных состояний в зависимости от приложенных деформаций для частиц различных размеров. Если при приложении деформации тип основного состояния изменяется, значит можно ожидать, что такие деформации могут привести к смене распредения намагниченности частицы. Мы изучили магнитные состояния эллиптических частиц с различными длинами осей, но при сохранении аспектного соотношения. Величины энергий различных состояний приве- дены в табл. 1, 2 и 3 для недеформированной и сжатой вдоль короткой или длинной оси частиц. Энергии основных состояний выделены жирным, прочерк означает, что данное состояние не реализуется. У недеформированных частиц большого размера основное состояние одновихревое, но с уменьшением размера анизотропия формы начинает давать больший вклад, и для частиц с длинной осью $200 \mathrm{~nm}$ основным уже становится квазиоднородное состояние. При деформациях сжатия вдоль длинной оси однородное состояние вдоль этой оси становится основным при любых размерах частиц. В сжатых вдоль короткой оси частицах возникает метастабильное квазиоднородное состояние вдоль короткой оси при больших размерах частицы, а также метастабильное двухвихревое состояние. При этом для любого размера основным является одновихревое состояние.

В табл. 4 показаны возможные переходы под воздействием деформаций для эллиптических $\mathrm{Ni}$ частиц. В таблице представлены три размера частиц и два типа состояний - квазиоднородное вдоль длинной оси и вихревое. Как видно из предыдущих таблиц, именно эти состояния всегда являются основыми. Жирным шрифтом показано более выгодное состояние. При больших размерах частицы возможен переход из вихревого состояния, которое является основным в недеформированной частице, в однородное состояние вдоль длинной оси, в результате сжатия вдоль этой оси. Так как одрородное состояние является метастабильным, то можно ожидать, что при „выключении“ деформаций частица останется в однородном состоянии. При этом, сжатием частиц вдоль короткой оси, можно всегда вернуться к вихревому состоянию. Таким образом, можно осуществлять переключение между различными состояниями. Схожая ситуация наблюдается в частицах $600 \times 300 \mathrm{~nm}$. В малых частицах $(200 \times 100 \mathrm{~nm})$ ситуация обратная. В отсутствие деформаций частица находится в квазиоднородном состоянии. Сжатием вдоль короткой оси ее можно перевести в вихревое состояние.

\section{2. Квадратные частицы}

Квадратная $\mathrm{Ni}$ частица со стороной $1 \mu \mathrm{m}$ и толщиной $30 \mathrm{~nm}$ может находиться в S-, C-состоянии и состоянии

Таблица 2. Энергии различных состояний для Ni эллиптической частицы с аспектным соотношением 2 к 1 и толщиной 30 nm, деформированной вдоль короткой оси. (Подчеркнуто и выделено жирным значение энергии основного состояния)

\begin{tabular}{|c|c|c|c|c|}
\hline \multirow{2}{*}{$\begin{array}{l}\text { Длинная ось } \\
\text { эллипса, } \mu \mathrm{m}\end{array}$} & \multicolumn{4}{|c|}{ Энергия состояния, $10^{-17}, J$} \\
\hline & $\begin{array}{c}\text { квазиоднородное } \\
\text { (длинная ось) }\end{array}$ & $\begin{array}{c}\text { квазиоднородное } \\
\text { (короткая ось) }\end{array}$ & одновихревое & двувихревое \\
\hline 1.0 & - & 12.8 & $\underline{7.1}$ & 7.5 \\
\hline 0.8 & 8.9 & - & $\underline{5.2}$ & 5.6 \\
\hline 0.6 & 6.1 & - & $\underline{3.5}$ & 3.9 \\
\hline 0.4 & 3.3 & - & $\underline{2.1}$ & 2.7 \\
\hline 0.2 & 1.1 & - & $\underline{1.0}$ & - \\
\hline
\end{tabular}



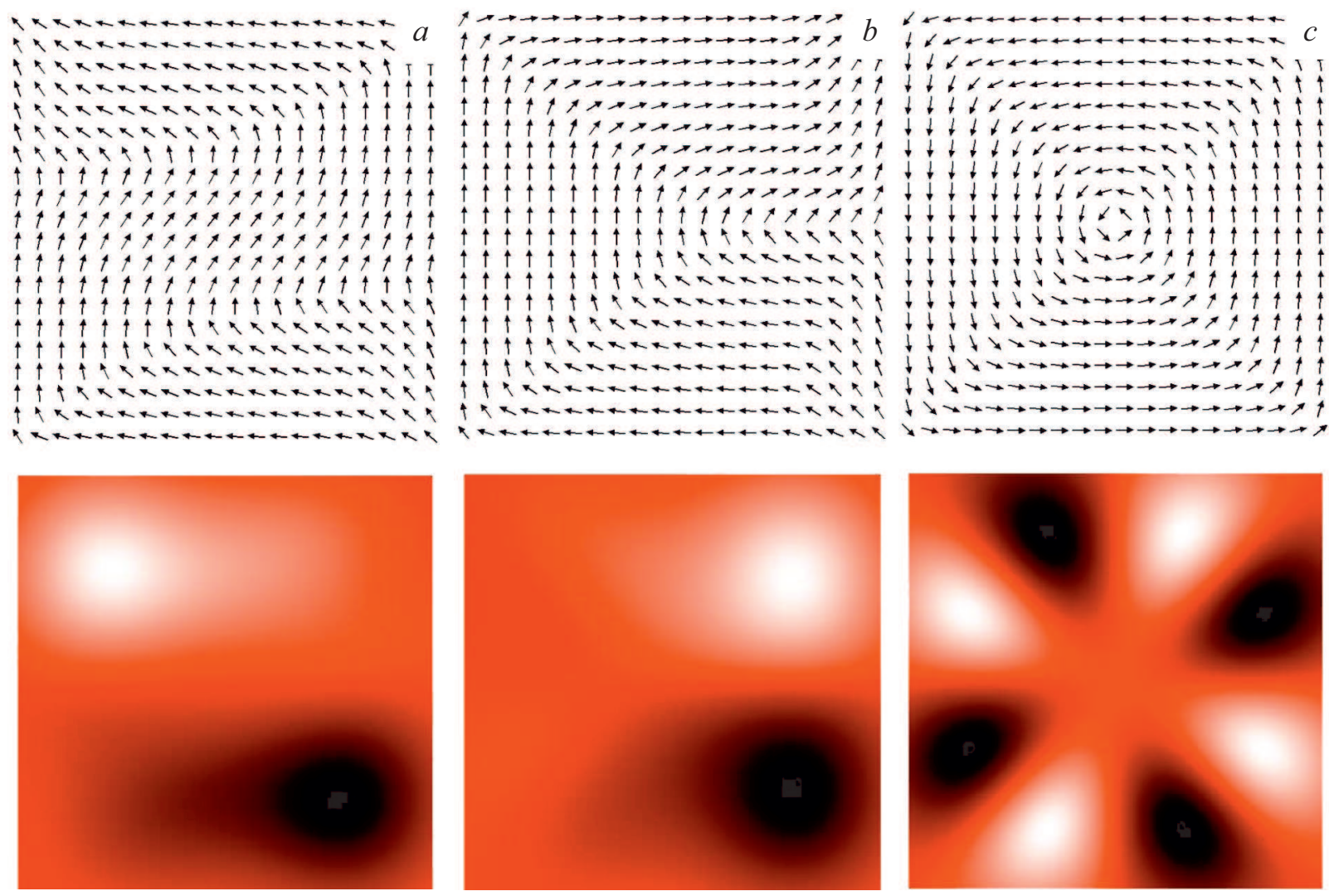

Рис. 3. Модельные распределения намагниченности и соответствующие им МСМ изображения квадратной Ni частицы с размером $1 \mu \mathrm{m}$ и толщиной $30 \mathrm{~nm}$ в S-состоянии $(a)$, С-состоянии $(b)$ и состоянии Ландау $(c)$ в отсутствие деформаций.

Ландау (см. рис. 3). Из-за отсутствия анизотропии в каком-либо из направлений и наличия острых углов квазиоднородное состояние в такой структуре не реализуется. Для деформации есть два выделенных направления - вдоль стороны и вдоль диагонали. При сжатии вдоль стороны S- и С-состояния переходят в квазиоднородное (рис. 4,a). Намагниченность состояния Ландау, как и вихрь в эллиптической частице, вытягивается вдоль оси деформации (рис. $4, b$ ), что можно наблюдать по МСМ изображению: контраст двух из четырех доменов стал сильнее за счет увеличения размеров этих доменов. Также под влиянием деформации возникло новое пятидоменное магнитное состояние (рис. 4,c). Такое состояние дает выигрыш в энергии магнитостатического взаимодействия по сравнению с C-состоянием. При сжатии по диагонали (рис. 5) S- и C-состояния вырождаются в квазиоднородное, а намагниченность в состоянии Ландау аналогично предыдущему случаю вытягивается вдоль направления деформации, что отражается на МСМ изображении. Во всех описанных случаях состояние Ландау является основным для частицы размером $1 \mu \mathrm{m}$. Таким образом, деформации не должны приводить к переходу из вихревого состония в квазиоднородное.

Мы определили полную энергию основных и метастабильных состояний как в отсутствие деформаций, так
Таблица 3. Энергии различных состояний для Ni эллиптической частицы с аспектным соотношением 2 к 1 и толщиной $30 \mathrm{~nm}$, деформированной вдоль длинной оси. (Подчеркнуто и выделено жирным значение энергии основного состояния)

\begin{tabular}{c|c|c}
\hline \multirow{2}{*}{$\begin{array}{c}\text { Длинная ось } \\
\text { эллипса, } \mu \mathrm{m}\end{array}$} & \multicolumn{2}{|c}{ Энергия состояния, $10^{-17}, J$} \\
\cline { 1 - 3 } & $\begin{array}{c}\text { квазиоднородное } \\
\text { (длинная ось) }\end{array}$ & одновихревое \\
\hline 1.0 & $\underline{\mathbf{5 . 4}}$ & 5.6 \\
0.8 & $\underline{\mathbf{4 . 1}}$ & 4.3 \\
0.6 & $\underline{\mathbf{2 . 9}}$ & 3.1 \\
0.4 & $\underline{\mathbf{1 . 8}}$ & 2.0 \\
0.2 & $\underline{\mathbf{0 . 7}}$ & 1.1
\end{tabular}

и при их наличии для квадратной частицы. Энергия определялась для частиц с длинами сторон $200 \mathrm{~nm}$, $600 \mathrm{~nm}$ и $1 \mu \mathrm{m}$. Величины энергий различных состояний приведены в табл. 5, 6 и 7 для недеформированной и сжатой вдоль стороны или диагонали частиц. Во всех случаях основным для квадратной частицы является состояние Ландау.

Наиболее привлекательным с точки зрения приложений является переключение между квазиоднородными состояниями. Поэтому важен вопрос, можно ли сделать 

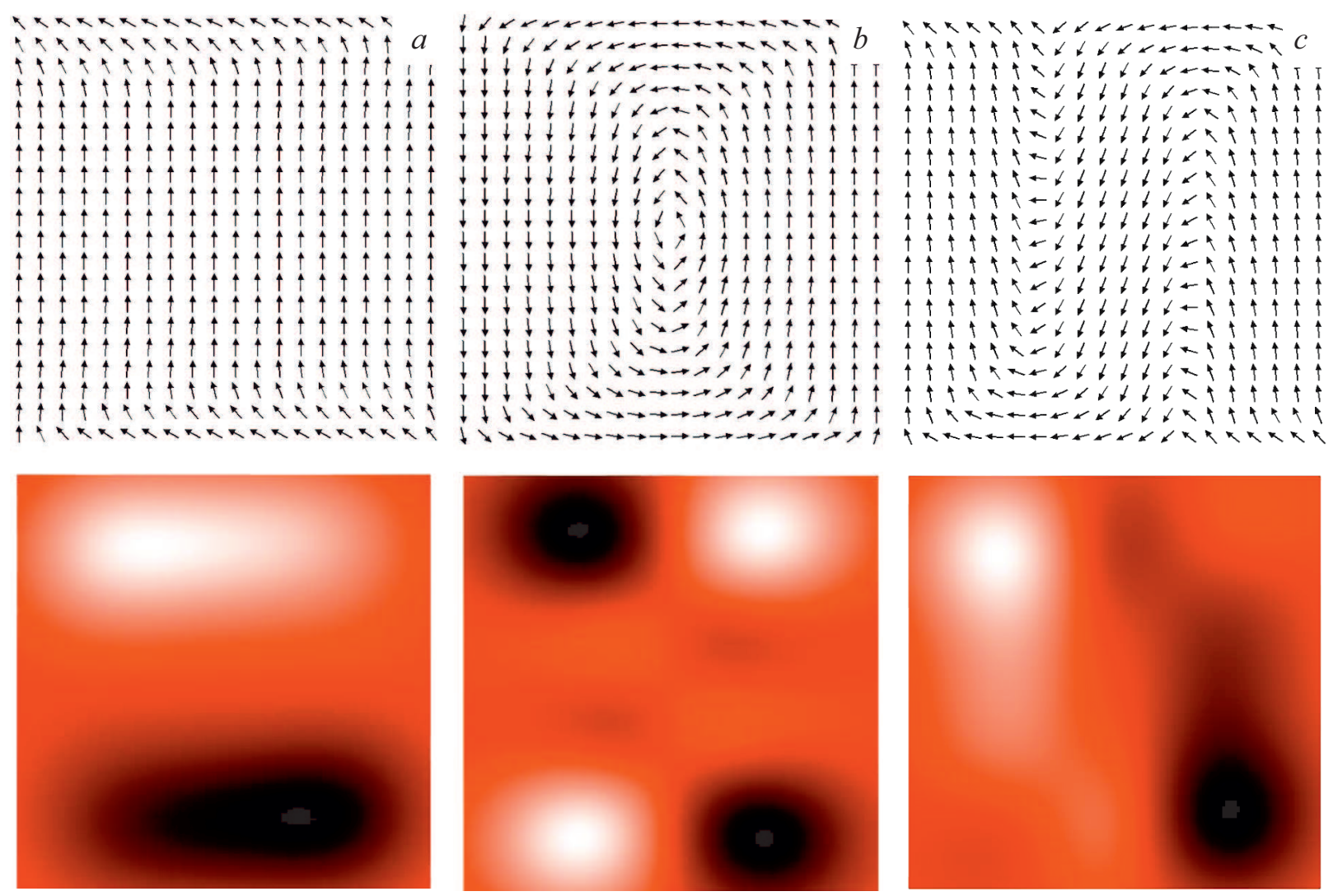

Рис. 4. Модельные распределения намагниченности и соответствующие им МСМ изображения квадратной Ni частицы с размером $1 \mu \mathrm{m}$ и толщиной $30 \mathrm{~nm}$ в квазиоднородном состоянии $(a)$, состоянии Ландау $(b)$ и пятидоменном состоянии $(c)$ при сжатии вдоль стороны.

так, что состояние Ландау не будет реализовываться в квадратной частице. В этом случае можно было бы говорить о переключении между квазиоднородными состояниями. Известно, что с уменьшением размеров магнитных частиц это состояние пропадает, и остается только квазиоднородное. Мы исследовали энергии распределений намагниченности в квадратных частицах в зависимости от их размеров и толщины и определи- ли, при каких размерах состояние Ландау в частице пропадает. В табл. 8 приведены максимальные размеры частицы, при которых оно не может быть реализовано. Расчеты показали, что эти размеры очень малы даже при толщине $10 \mathrm{~nm}$ сторона квадрата не должна превышать $145 \mathrm{~nm}$. В частицах с размерами меньше критических реализуются только квазиоднородные состояния. При этом в силу симметрии частицы будут

Таблица 4. Переключение между магнитными состояними эллиптической частицы с соотношением осей 2 к 1 при ее деформировании. Энергия записана в единицах $J \cdot 10^{-17}$. (Подчеркнуто и выделено жирным значение энергии основного состояния

\begin{tabular}{c|c|c|c|c|c|c}
\hline Длина оси & \multicolumn{2}{|c|}{1000 нм } & \multicolumn{2}{c|}{600 нм } & \multicolumn{2}{c}{200 нм } \\
\hline Состояние & $\begin{array}{c}\text { Квазиоднородное } \\
\text { вдоль длинной оси }\end{array}$ & Вихревое & $\begin{array}{c}\text { Квазиоднородное } \\
\text { вдоль длинной оси }\end{array}$ & Вихревое & $\begin{array}{c}\text { Квазиоднородное } \\
\text { вдоль длинной оси }\end{array}$ & Вихревое \\
\hline $\begin{array}{c}\text { Нет } \\
\text { деформации }\end{array}$ & 8.287 & $\underline{\mathbf{6 . 9 5 6}}$ & 4.117 & $\underline{\mathbf{3 . 4 9 5}}$ & $\underline{\mathbf{0 . 8 8 1}}$ & 1.107 \\
\hline $\begin{array}{c}\text { Сжатие вдоль } \\
\text { длинной оси }\end{array}$ & $\underline{\mathbf{5 . 3 9 4}}$ & 5.606 & $\underline{\mathbf{2 . 9 3 1}}$ & 3.102 & $\underline{\mathbf{0 . 7 2 6}}$ \\
\hline $\begin{array}{c}\text { Сжатие вдоль } \\
\text { короткой оси }\end{array}$ & - & $\underline{\mathbf{6 . 9 5 6}}$ & 6.164 & $\underline{\mathbf{3 . 4 8 8}}$ & 1.098 \\
\hline
\end{tabular}


Таблица 5. Энергии различных состояний для недеформированной $\mathrm{Ni}$ квадратной частицы толщиной $30 \mathrm{~nm}$. (Подчеркнуто и выделено жирным значение энергии основного состояния)

\begin{tabular}{c|c|c|c}
\hline \multirow{2}{*}{$\begin{array}{c}\text { Сторона } \\
\text { квадрата, } \mu \mathrm{m}\end{array}$} & \multicolumn{3}{|c}{ Энергия состояния, $10^{-17}, \mathrm{~J}$} \\
\cline { 2 - 4 } & $S$-состояние & $C$-состояние & $\begin{array}{c}\text { состояние } \\
\text { Ландау }\end{array}$ \\
\hline 1.0 & 9.9 & 9.2 & $\underline{\mathbf{2 . 7}}$ \\
0.6 & 6.0 & 5.5 & $\underline{\mathbf{1 . 9}}$ \\
0.2 & 1.9 & 1.7 & $\underline{\mathbf{1 . 0}}$
\end{tabular}

Таблица 6. Энергии различных состояний для Ni квадратной частицы толщиной $30 \mathrm{~nm}$, деформированной вдоль стороны. (Подчеркнуто и выделено жирным значение энергии основного состояния)

\begin{tabular}{c|c|c|c}
\hline \multirow{2}{*}{$\begin{array}{c}\text { Сторона } \\
\text { квадрата, } \mu \mathrm{m}\end{array}$} & \multicolumn{3}{|c}{ Энергия состояния, $10^{-17} \mathrm{~J}$} \\
\cline { 2 - 4 } & $\begin{array}{c}\text { квазиоднородное } \\
\text { состояние }\end{array}$ & $\begin{array}{c}\text { состояние } \\
\text { Ландау }\end{array}$ & $\begin{array}{c}\text { пятидоменное } \\
\text { состояние }\end{array}$ \\
\hline 1.0 & 15.1 & $\underline{\mathbf{1 1 . 7}}$ & 19.4 \\
0.6 & 8.0 & $\underline{\mathbf{5 . 6}}$ & - \\
0.2 & 2.0 & $\underline{\mathbf{1 . 5}}$ & - \\
\hline
\end{tabular}

Таблица 7. Энергии различных состояний для Ni квадратной частицы толщиной $30 \mathrm{~nm}$, деформированной вдоль диагонали. (Подчеркнуто и выделено жирным значение энергии основного состояния)

\begin{tabular}{c|c|c}
\hline \multirow{2}{*}{$\begin{array}{c}\text { Сторона } \\
\text { квадрата, } \mu \mathrm{m}\end{array}$} & \multicolumn{2}{|c}{ Энергия состояния, $10^{-17} \mathrm{~J}$} \\
\cline { 2 - 3 } & $\begin{array}{c}\text { квазиоднородное } \\
\text { состояние }\end{array}$ & $\begin{array}{c}\text { состояние Ландау } \\
\text { (вихревое) }\end{array}$ \\
\hline 1.0 & 13.4 & $\underline{\mathbf{1 1 . 5}}$ \\
0.6 & 7.3 & $\underline{\mathbf{5 . 6}}$ \\
0.2 & 2.0 & $\underline{\mathbf{1 . 5}}$
\end{tabular}

2 эквивалентные ортогональные оси, вдоль которых может быть направлена намагниченность. При этом возможно переключение между этими состояниями за счет магнитоэлектрического эффекта. Однако наблюдение таких частиц с помощью магнитно-силового микроскопа является проблематичным, поскольку $\mathrm{Ni}$ имеет маленькую намагниченность и дает слабый контраст.

Таким образом, переключение между различными состояниями в квадратных частицах $\mathrm{Ni}$, схожее с описанным для эллиптических частиц, по-видимому, очень трудно реализовать на практике.

\section{3. Треугольные частицы}

Мы исследовали треугольные частицы с длинами сторон $200 \mathrm{~nm}, 600 \mathrm{~nm}$ и $1 \mu \mathrm{m}$ и толщиной $30 \mathrm{~nm}$. В отсутствие деформаций в таких частицах может реализовываться либо двухдоменное состояние (рис. $6, a$ ), либо вихревое (см. рис. $6, b$ ). Важно отметить, что в силу симметрии частицы есть три пары эквивалентных двухдоменных состояний с различной ориентацией среднего магнитного момента. Для треугольной частицы есть два выделенных направления деформаций — вдоль стороны
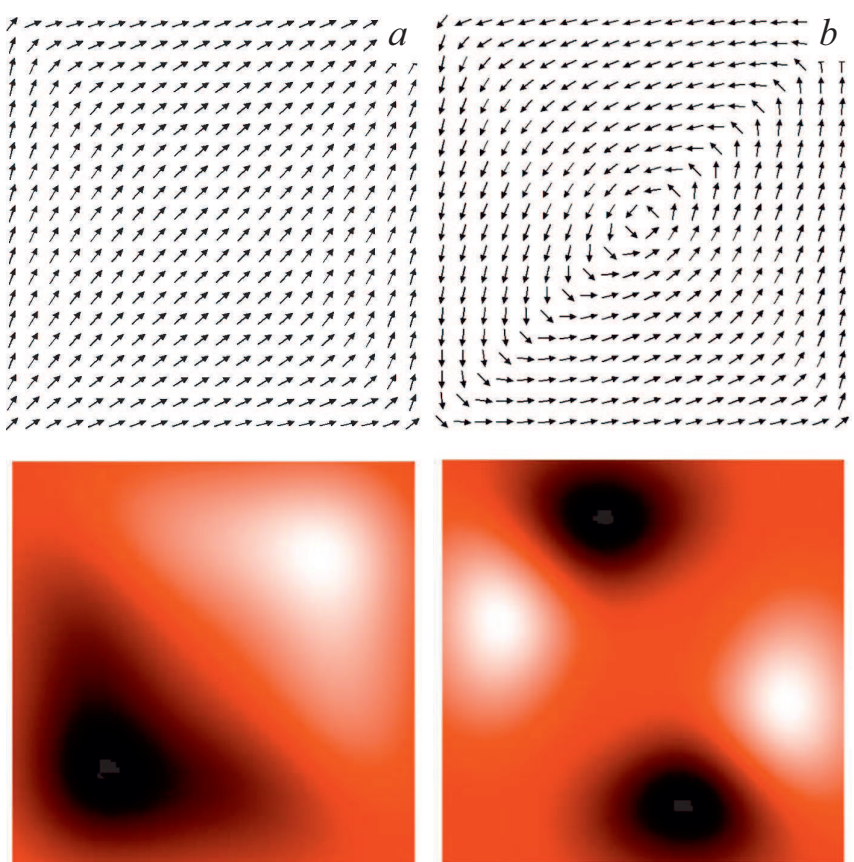

Рис. 5. Модельные распределения намагниченности и соответствующие им МСМ изображения квадратной Ni частицы с размером $1 \mu \mathrm{m}$ и толщиной $30 \mathrm{~nm}$ в квазиоднородном $(a)$ и Ландау $(b)$ состояниях при сжатии вдоль диагонали.

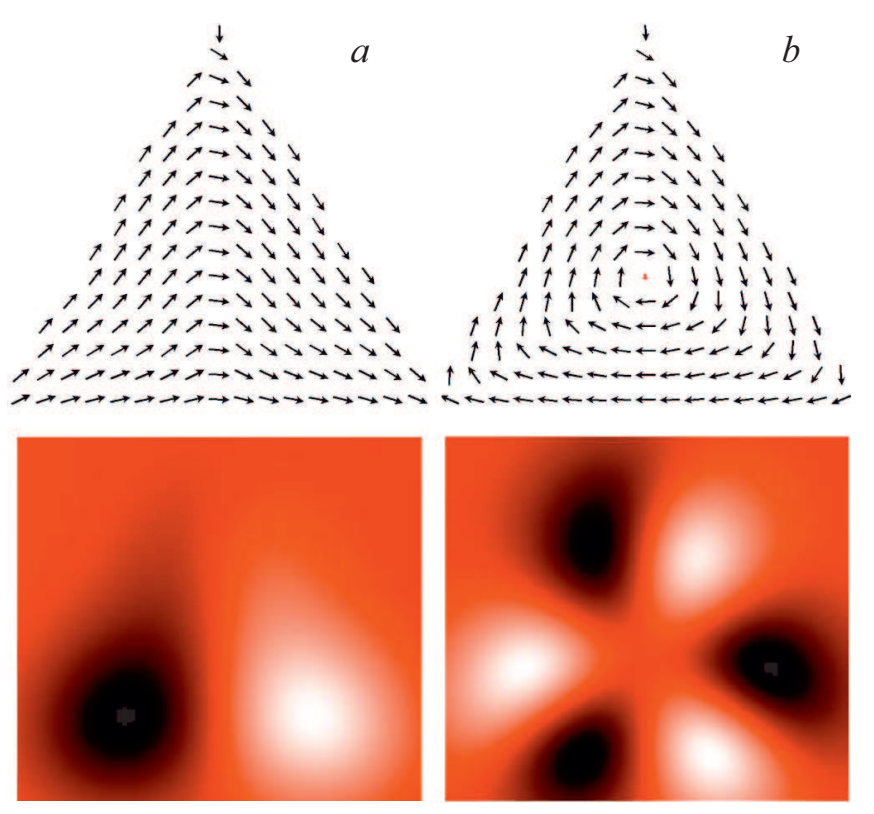

Рис. 6. Модельные распределения намагниченности и соответствующие им МСМ изображения треугольной Ni частицы с размером $1 \mu \mathrm{m}$ и толщиной $30 \mathrm{~nm}$ в двухдоменном $(a)$, вихревом $(b)$ состояниях в отсутствие деформаций. 


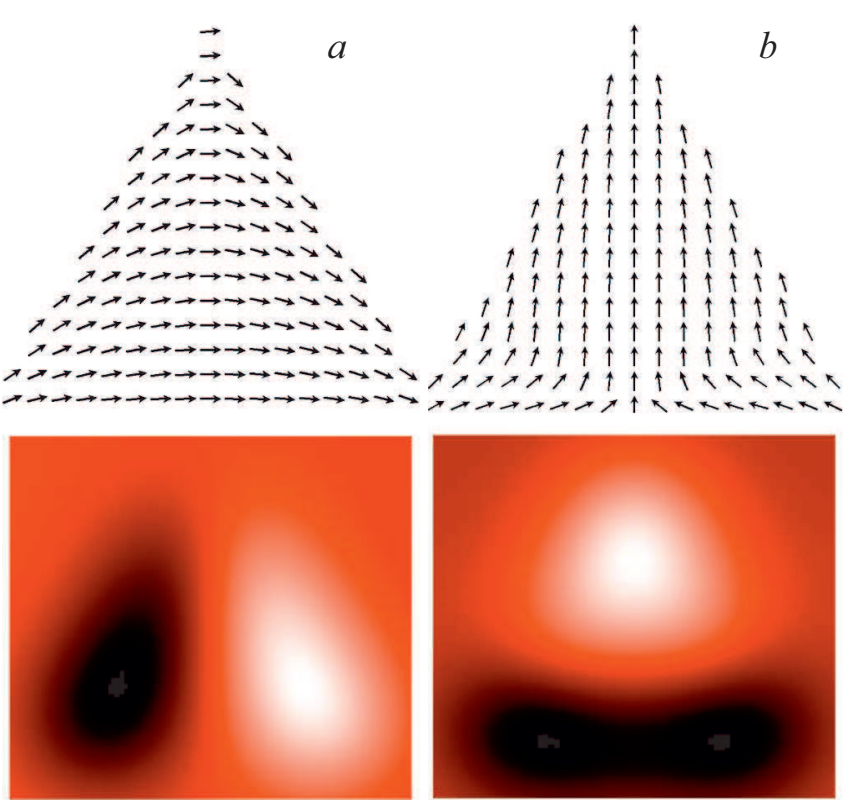

Рис. 7. Модельные квазиоднородные распределения намагниченности и соответствующие им МСМ изображения треугольной $\mathrm{Ni}$ частицы с размером $1 \mu \mathrm{m}$ и толщиной $30 \mathrm{~nm}$ при сжатии вдоль стороны $(a)$ и вдоль биссектрисы $(b)$.

и вдоль биссектрисы. Сжатие в указанных направлениях добавляет новые метастабильные состояния квазиоднородные со средней намагниченностью вдоль указанных направлений (рис. 7). Величины энергий
Таблица 8. Максимальный размер Ni квадратной частицы, при котором вихревое состояние в ней не реализуется, для различных толщин этой частицы

\begin{tabular}{l|c|c|c|c|c}
\hline Толщина, $\mathrm{nm}$ & 10 & 20 & 30 & 40 & 50 \\
\hline Сторона, $\mathrm{nm}$ & 145 & 110 & 110 & 110 & 100
\end{tabular}

Таблица 9. Энергии различных состояний для недеформированной Ni треугольной частицы толщиной $30 \mathrm{~nm}$ (Подчеркнуто и выделено жирным значение энергии основного состояния)

\begin{tabular}{c|c|c}
\hline \multirow{2}{*}{$\begin{array}{c}\text { Сторона } \\
\text { квадрата, } \mu \mathrm{m}\end{array}$} & \multicolumn{2}{|c}{ Энергия состояния, $10^{-17} \mathrm{~J}$} \\
\cline { 2 - 3 } & $\begin{array}{c}\text { двухдоменное } \\
\text { состояние }\end{array}$ & $\begin{array}{c}\text { вихревое } \\
\text { состояние }\end{array}$ \\
\hline 1.0 & 5.5 & $\underline{\mathbf{4 . 0}}$ \\
0.6 & 3.2 & $\underline{\mathbf{2 . 7}}$ \\
0.2 & $\underline{\mathbf{1 . 0}}$ &
\end{tabular}

различных состояний приведены в табл. 9, 10 и 11 для недеформированной и сжатой вдоль стороны или биссектрисы или частиц.

В больших частицах $(1000 \mathrm{~nm})$ вихревое состояние является основным и при наличии, и в отсутствие деформаций. Поэтому переключение между состояниями за счет деформаций невозможно в таких частицах.

В малых частицах (600 nm и $200 \mathrm{~nm})$ ситуация другая (рис. 8). Если треугольную частицу, находящуюся в
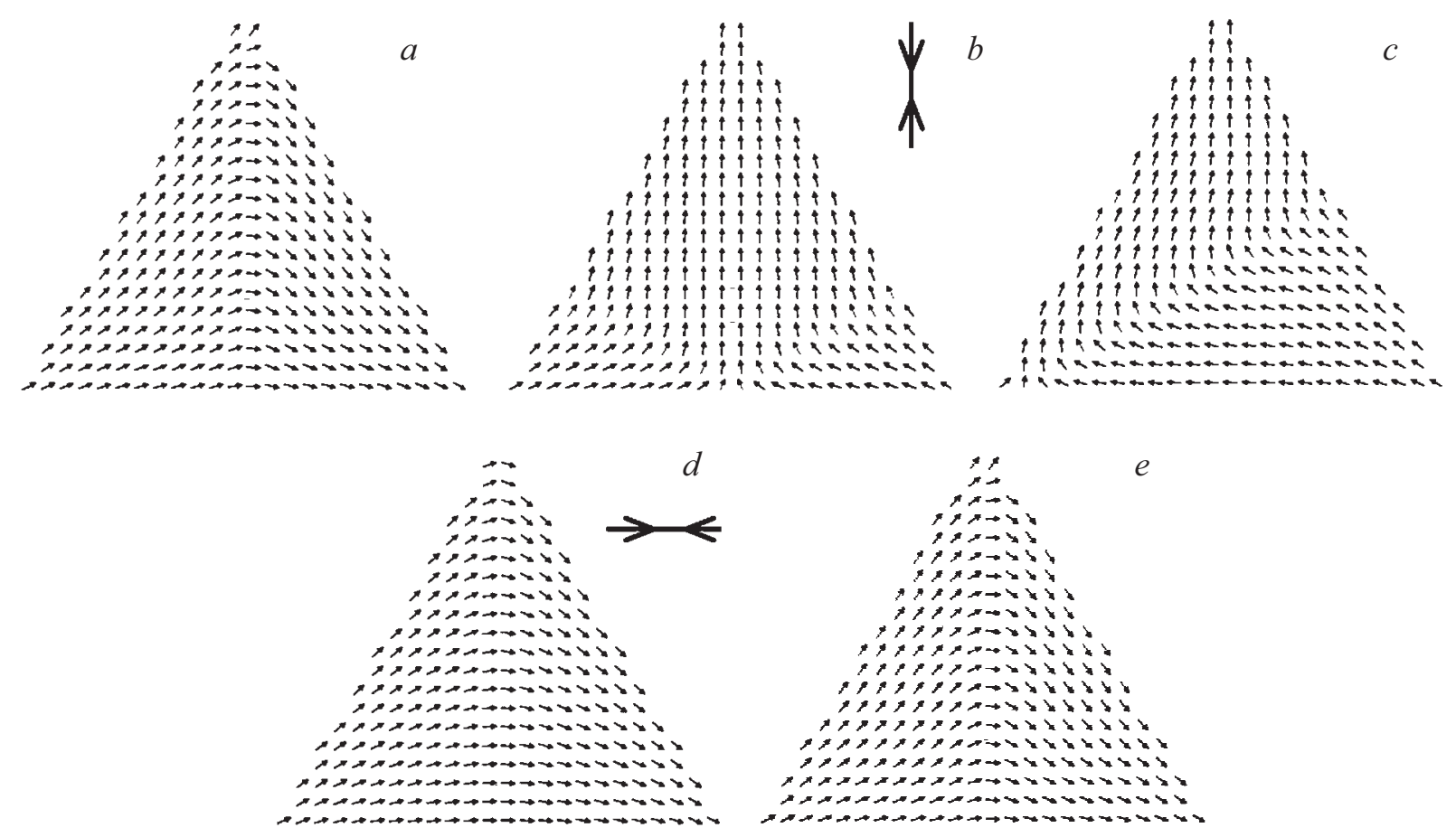

Рис. 8. Переключение состояний треугольной частицы со стороной $600 \mathrm{~nm}$. $a$ - деформации отсутствуют, $b-$ частица сжимается вдоль биссектрисы, $c$ - деформация снимается, $d$ - частица сжимается вдоль стороны, $e$ - деформация снимается. 
Таблица 10. Энергии различных состояний для $\mathrm{Ni}$ треугольной частицы толщиной $30 \mathrm{~nm}$, деформированной вдоль стороны. (Подчеркнуто и выделено жирным значение энергии основного состояния

\begin{tabular}{c|c|c}
\hline \multirow{2}{*}{$\begin{array}{c}\text { Сторона } \\
\text { квадрата, } \mu \mathrm{m}\end{array}$} & \multicolumn{2}{|c}{ Энергия состояния, $10^{-17} \mathrm{~J}$} \\
\cline { 2 - 3 } & $\begin{array}{c}\text { квазиоднородное } \\
\text { состояние }\end{array}$ & $\begin{array}{c}\text { вихревое } \\
\text { состояние }\end{array}$ \\
\hline 1.0 & 8.0 & $\underline{\mathbf{7 . 6}}$ \\
0.6 & 4.2 & $\underline{\mathbf{4 . 1}}$ \\
0.2 & $\underline{\mathbf{1 . 1}}$ & $\mathbf{1 . 3}$
\end{tabular}

Таблица 11. Энергии различных состояний для Ni треугольной частицы толщиной $30 \mathrm{~nm}$, деформированной вдоль биссектрисы. (Подчеркнуто и выделено жирным значение энергии основного состояния)

\begin{tabular}{c|c|c|c}
\hline \multirow{2}{*}{$\begin{array}{c}\text { Сторона } \\
\text { квадрата, } \mu \mathrm{m}\end{array}$} & \multicolumn{3}{|c}{ Энергия состояния, $10^{-17} \mathrm{~J}$} \\
\cline { 2 - 4 } & $\begin{array}{c}\text { квазиоднородное } \\
\text { состояние }\end{array}$ & $\begin{array}{c}\text { двухдоменное } \\
\text { состояние }\end{array}$ & $\begin{array}{c}\text { вихревое } \\
\text { состояние }\end{array}$ \\
\hline 1.0 & 8.2 & 10.1 & 8.1 \\
0.6 & 4.2 & 5.2 & 4.3 \\
0.2 & 1.1 & - & 1.3
\end{tabular}

двухдоменном состоянии со средней намагниченностью вдоль основания (рис. 8,a), сжать по биссектрисе, вдоль которой расположена доменная стенка, то частица переходит в квазиоднородное состояние (рис. $8, b$ ), намагниченное вдоль оси сжатия. После снятия деформации частица не остается в квазиоднородном состоянии, а переходит в двухдоменное (рис. 8,c), но в этом случае средняя намагниченность будет направлена уже не вдоль основания, а вдоль одной из сторон треугольника. Чтобы перевести частицу в исходное состояние, необходимо сжать ее вдоль основания (рис. 8, $d$ ), а затем убрать деформацию (рис. 8,e). Таким образом, в малых треугольных частицах есть возможность осуществлять переключение магнитного состояния за счет деформаций.

\section{4. Заключение}

Таким образом, в работе представлены результаты моделирования влияния механических деформаций на магнитное состояние в субмикронных частицах квадратной, эллиптической и треугольной форм. Показано, что деформация может как изменить основное состояние, так и увеличить число возможных метастабильных состояний. Кроме того, механическая деформация позволяет переключать состояние частиц. В эллиптической частице с аспектным соотношением 1/2 возможно переключение между квазиоднородным и вихревым состояниями. В треугольной частице возможно переключение между двумя двухдоменными состояниями с различной ориентацией средней намагниченности. В квадратной частице получить переключение между различными состояниями нам не удалось.

\section{Финансирование работы}

Работа выполнена при поддержке РНФ № 18-72-10026.

\section{Конфликт интересов}

Авторы заявляют, что у них нет конфликта интересов.

\section{Список литературы}

[1] Greg P. Carman. IEEE Transact. Magn. 51, 0600104 (2015).

[2] Manfred Fiebig. J. Phys. D: Appl. Phys. 38, R123 (2005).

[3] S. Fusil, V. Garcia, A. Barthelemy, M. Bibes. Ann. Rev. Mater. Res. 44, 91 (2014).

[4] W. Eerenstein, N.D. Mathur, J.F. Scott. Nature 442, 759 (2006).

[5] R. Ramesh, N.A. Spaldin. Nature Mater. 6, 21 (2007).

[6] M. Bibes, A. Barthelemy. Nature Mater. 7, 425 (2008).

[7] H. Ohno, D. Chiba, F. Matsukura, T. Omiya, E. Abe, T. Dietl, Y. Ohno, K. Ohtani. Nature 408, 944 (2000).

[8] S. Geprags, A. Brandlmaier, M. Opel, R. Gross, S.T.B. Goennenwein. Appl. Phys. Lett. 96, 142509 (2010).

[9] R. Sreubel, D. Kohler, R. Schafer, L.M. Eng. Phys. Rev. B 87, 054410 (2013).

[10] M. Weiler, A. Brandlmaier, S. Geprags, M. Althammer, M. Opel, C. Bihler, H. Huebl, M.S. Brandt, R. Gross, S.T.B. Goennenwein. New J. Phys. 11, 013021 (2009).

[11] R.V. Chopdekar, Y. Suzuki. Appl. Phys. Lett. 89, 182506 (2006).

[12] M. Ziese, A. Bollero, I. Panagiotopoulos, N. Moutis. Appl. Phys. Lett. 88, 212502 (2006).

[13] S.W. Fackler, M.J. Donahue, T. Gao, P.N.A. Nero, S.W. Cheong, J. Cumings, I. Takeuchi. Appl. Phys. Lett. 105, 212905 (2014).

[14] R. Streubel, D. Kohler, R. Schafer, L.M. Eng. Phys. Rev. B 87, 054410 (2013).

[15] T. Taniyama. J. Phys.: Condens. Matter 27, 504001 (2015).

[16] M. Buzzi, R.V. Chopdekar, J.L. Hockel, A. Bur, T. Wu, N. Pilet, P. Warnicke, G.P. Carman, L.J. Heyderman, F. Nolting. Phys. Rev. Lett. 111, 027204 (2013).

[17] M. Salehi-Fashami, N. D’Souza. J. Magn. Magn. Mater. 438, 76 (2017).

[18] K. Nanayakkara, I.S. Vasil'evskii, I.S. Eremin, O.S. Kolentsova, N.I. Kargin, A. Anferov, A. Kozhanov. J. Appl. Phys. 119, 233906 (2016).

[19] B. Lambson, Z. Gu, M. Monroe, S. Dhuey, A. Scholl, J. Bokor. Appl. Phys. A 111, 413 (2013).

[20] Д.А. Бизяев, А.А. Бухараев, А.П. Чукланов, Н.И. Нургазизов. ФТТ 60, 2152 (2018). 
[21] А.А. Бухараев, А.К. Звездин, А.П. Пятаков, Ю.К. Фетисов. УФН 188, 1288 (2018).

[22] I. Gilbert, A.C. Chavez, D.T. Pierce, J. Unguris, W.-Y. Sun, C.Y. Liang, G.P. Carman. Appl. Phys. Lett. 109, 162404 (2016).

[23] M. Buzzi, R.V. Chopdekar, J.L. Hockel, A. Bur, T. Wu, N. Pilet, P. Warnicke, G.P. Carman, L.J. Heyderman, F. Nolting. Phys. Rev. Lett. 111, 027204 (2013).

[24] M.J. Donahue, D.G. Porter. Interagency Report NISTIR 6376. National Institute of Standards and Technology, Gaithersburg; http://math.nist.gov/oommf/

[25] В.Л. Миронов. Основы сканирующей зондовой микроскопии. Техносфера, М. (2009). 144 с.

Редактор Ю.Э. Китаев 